\title{
Preserving spatial and temporal dimensions in observational data of segregation
}

\section{Colin Tredoux*}

Department of Psychology, University of Cape Town, Private Bag, Rondebosch 7701, South Africa.

e-mail: plato@humanities.uct.ac.za

\section{John Dixon}

Department of Psychology, Lancaster University, United Kingdom

\section{Stephen Underwood ${ }^{1}$}

Department of Psychology, University of Cape Town, South Africa

\section{David Nunez}

Department of Psychology, University of Cape Town, South Africa

\section{Gillian Finchilescu}

Department of Psychology, University of the Witwatersrand, Johannesburg, South Africa

*To whom correspondence should be addressed

Recent approaches to the study of intergroup contact have emphasised the need for naturalistic studies and the importance of paying attention to the spatiality of contact. In this article it is argued that it is important to preserve both spatiality and temporality when studying inter-group contact in naturalistic settings. This is not easy to do with existing observational methods, and a novel approach is proposed. Photographs are taken of a public space with a fixed periodicity and vantage point, and with knowledge of the physical layout of the space, three-dimensional, time-marked data points are recorded for each inhabitant. A public space on a university campus was used as a test bed, and data are reported that show it to be very useful, giving fresh insights into the nature of segregation and 
integration in informal leisure spaces, as well as providing evidence of the importance of taking temporality into account when studying naturalistic instances of inter-group contact.

Keywords: contact hypothesis; inter-group contact; naturalistic observation; spatiality; temporality

People from different social groups frequently come into contact with one another. They may attempt to avoid contact by inhabiting different spaces, or, when obliged to share the same physical space, they may structure their temporal use of it so that they are unlikely to encounter members of other groups (Dixon \& Durrheim, 2003). However, this spatial and temporal structuration of intergroup contact is never completely successful. The failure of 'Grand Apartheid', one of the most extreme of such attempts, is a clear demonstration of this (Christopher, 1991).

What is the consequence of the inevitable contact between social groups? This question is one of the most enduring and significant in social psychology, and the conjectural answer to it is widely known as the contact hypothesis. The hypothesis states that contact between members of social groups may lead to a reduction in levels of prejudice against one another. Social psychologists have expended a great deal of effort on this hypothesis. Pettigrew and Tropp (2000) report a meta-analysis of over 200 empirical contact studies, published over a period of 60 years, and conducted in a range of countries (including South Africa, see Mynhardt \& du Toit, 1991, for a review). The conclusion Pettigrew and Tropp draw is fairly widely accepted now, namely that contact between members of social groups can reduce levels of prejudice and ill-will. However, this reduction will only occur under certain conditions, and if these conditions are absent, levels of prejudice may increase rather than decrease (Pettigrew, 1998).

There are many problems with the way social psychology has conceptualised and studied intergroup 'contact'. It is not our concern here to provide a detailed discussion of, and justification for, this claim, as several authors have already done this (cf. Dixon and Reicher, 1997). We do wish to point out that intergroup contact has been conceptualised in a decontextualised manner in social psychology, and this has had several consequences. In the first place, the 'contact' in much of this research occurs under rather artificial and idealised circumstances. Typically, contact is manipulated in laboratories to have certain characteristics, or is observed in quasi-experimental settings. There is very little research that examines contact as a behavioural phenomenon, occurring in natural settings. Research that has addressed contact as a naturally occurring behavioural form, on the other hand, has usually found that contact rarely conforms to that hypothesised in the literature of 'ideal conditions'. What appears to emerge from contact in these settings, instead, are various forms of segregation and racialisation. Studies have generally come to a sobering conclusion, namely that the predominant way of organising intergroup relations is segregationist (e.g. Schofield \& Sagar, 1977; Taylor \& Moghaddam, 1996). At a larger, macro-social level, this is 
exactly what appears to have happened in the United States of America (US), where contact was engineered over a long period in an attempt to produce better inter-group and race relations, but that country remains fundamentally segregated (Goldberg, 1998).

In addition to the theoretical impoverishment of contact research, there has been a general neglect of field studies in the contact literature, thus failing to document instances of naturally occurring contact (Stephan, 1987). Some field studies have been conducted in residential and educational settings in the US, though, and despite the absence of 'ideal contact conditions', they report some positive findings. Jahoda and West (1951), for instance, found that people in an interracial housing estate who lived in integrated housing blocks were more likely to be in favour of interracial housing than those who lived in segregated blocks.

More relevant to the present article are those studies that have made direct observations of contact or physical proximity. These studies typically collected data about seating patterns in ostensibly integrated contexts. Campbell, Kruskal and Wallace (1966) analysed seating patterns in three desegregated schools and found that children tended to congregate in homogeneous racial groups to a greater extent than would be expected by chance or random variation. Davis, Seibert and Breed (1966) studied seating patterns in New Orleans public buses and trams, following the desegregation of public transport in 1958. They noted a relatively low level of integration. Schofield and Sagar (1977) examined seating patterns in a school cafeteria and found support for the contact hypothesis, since seating patterns became more integrated over time in the seventh grade (where there were optimal conditions for contact) but integration decreased in the eighth grade (where conditions for contact were less optimal). Schofield and Francis (1982) found that race was a strong factor in predicting interaction between white and black schoolchildren in classroom settings, and in a follow-up study, Sagar, Schofield and Snyder (1983) found that race (along with gender) was a significant grouping variable when studying student interactions in a desegregated classroom setting. These studies showed some support for the contact hypothesis in naturally occurring settings, but race segregation was nevertheless still very evident.

Other field studies have produced results that are less supportive of the contact hypothesis. Rogers, Hennigan, Bowman and Miller (1984) conducted an observational field study in which they attempted to analyse the effects that interracial co-operative activities could have in a school playground setting. The intervention appeared to have little impact, but the co-presence of many unexpected confounding factors made alternative interpretations difficult to reject. Henderson-King (1994) found that subtle prejudice increased in a neutral interracial contact encounter immediately following proximal contact of an unfavourable nature (witnessing an argument between a black couple). 


\section{CONTACT AND THE SPATIAL ORGANISATION OF EVERYDAY RELATIONS}

If the problem of (re)segregation is a pertinent one for the study of contact research outside the laboratory, then researchers neglect spatial considerations at their peril. Dixon (2001) calls for contact researchers to acknowledge the role that spatial boundaries play in mediating contact. He argues that space should not be perceived as a passive backdrop to group relations, but 'as a meaningful and dynamic production that constitutes our collective relations and identities' (Dixon, 2001, p. 587).

Goldberg (1993) argues that racism becomes established when spatial structures are normalised according to racialised discourse, so that they seem natural. Dixon and Reicher (1997) highlight this in their study of the discourse of white residents in the Cape Town suburb of Hout Bay after a recent award of land to a predominantly black group of people. It is not that space has necessarily been ignored in contact studies, rather that it has not occupied importance in explanatory models. For instance, Jahoda and West (1951) noted that community laundry rooms located in the basement of interracial housing units were the site of greater intergroup friendship establishment than a community facility building that had been established to promote interracial friendship. However, rather than attribute spatial significance to this remarkable finding they explained it according to informal as opposed to formal contact. Similarly, Davis, Seibert and Breed (1966) note how they frequently observed white and black women talking at the bus stop only to separate upon boarding the bus, and rather than understanding this in the light of the historical associations of 'bus space' as opposed to 'bus stop space' they attribute it to the general ambience created by public transportation. Rogers et al. (1986) partially explain the failure of their playground activity contact intervention by stating that the activities, which took place three times a week, required novel equipment and when this equipment was removed, the (structural or spatial) cue for cross-race interaction was also removed. However, they do not elaborate on what this might mean about spatial cues mediating contact.

Apart from a lack of overall consideration of space, there is also a gap in the literature regarding contact in leisure space in particular (Philipp, 2000). Since leisure and, consequently, leisure space is so intricately bound to free choice, it seems to be a fruitful area in which contact research could enhance our understanding of race relations (Philipp, 2000). Dixon and Durrheim's (2003) study of informal segregation on a KwaZulu-Natal beachfront represents an attempt to study contact in a leisure space, where spatial processes are elevated to significance. They conclude their study by suggesting that 'social psychologists need to pay closer attention to the varieties of informal segregation that shape inter-group relations in everyday life, seeking to uncover the practices, activities and forms of social organization that enable their reproduction' (Dixon \& Durrheim, p. 21). 


\section{CONTACT AND THE TEMPORAL ORGANISATION OF EVERYDAY RELATIONS}

We consider this theoretical interest in the spatiality of intergroup contact to be highly significant. However, we think that the theoretical framework needs to be re-dimensioned to take account of the temporal nature of intergroup contact and segregation. This is especially the case when the focus is on varieties of informal contact and segregation, and when the method of study is observational.

In the first place, sensitivity to the sequence in which an intergroup engagement unfolds can give us information about the active segregation of space in a way that the cross-sectional observation of a space cannot. A segregated seating pattern, for instance, can come about in a number of ways, some of which may have nothing to do with a desire to enforce physical intergroup distance (Schelling, 1978). Similarly, an apparently integrated seating pattern can come about even though groups would prefer to enforce a separation. This will probably happen in a venue where there is a shortage of available space, and actors in that space are obliged to choose whatever seat or position is available to them. A record of how a particular seating configuration assembles may reveal patterns that are hidden from cross-sectional observation. In the second of our examples above, a temporal record of the seating pattern may show that it started off in starkly segregated form and that this continued until the ratio of people to empty seats reached some critical index, much in the fashion of the 'critical mass' models discussed by Schelling (1978).

In the second place, a record of physical occupation over time allows finer-grained observations than can be obtained from a cross-sectional analysis. Individuals or groups of individuals can be tracked and their specific choice of physical position interrogated. For instance, a model of seating choice premised on the relative population density of the space and the relative proportions of group members can be tested on the basis of observations collected in a time sequence. It is doubtful whether this could be done with detemporalised observations of the same space. Detemporalised data may allow one to assert that a particular space is segregated along group lines, but it says little about how that segregation came about. If it is important to focus on informal processes of contact and segregation, that is, on the production and reproduction of segregation in the everyday, as Dixon and colleagues have argued (Dixon, 2001; Durrheim \& Dixon, 2001), then it must be important to collect data that preserves both the spatiality and temporality of behaviour.

\section{A METHODOLOGICAL FRAMEWORK FOR STUDYING THE SPATIAL AND TEMPORAL DIMENSIONS OF INTER-GROUP RELATIONS IN EVERYDAY SPACES}

In the present study we explore a methodology for collecting observations in a manner that preserves the spatial and temporal coordinates in observations of contact and segregation. The general procedure is to (a) take digital 
photographs of a target space with a specified periodicity, (b) code the two-dimensional spatial coordinates of each observation within each period, and (c) to transform the two-dimensional coordinates to three-dimensional coordinates using knowledge of the physical properties of the space. We use this methodology in the present case for studying seating patterns of students in a public space, but it could perhaps be used with little modification for other research investigations where spatial or temporal context is important.

As far as we are aware, the observational technique we propose is novel to the study of contact and segregation. Previous observational studies (many of which were discussed earlier) have used observers to count the number of people of various groupings, within semi-arbitrary spatial divisions (e.g., in rows within a classroom, or a bus). Few have attempted to record temporality. There is well-established literature regarding the statistical analysis of data of this kind, insofar as the observational situation is analogous to the methods used in urban geography (e.g., Massey \& Denton, 1988), and such analysis can reveal much about patterns of segregation or contact. For examples of studies that have explicitly emulated these methods, the reader is refered to McAuley, Plummer, Moskalenko and Mordkoff (2001), and Dixon and Durrheim (2003). However, as we argued above, methods that do not preserve temporality, or which use gross spatial partitions, are likely to elide important aspects of the empirical phenomena they study.

The particular space we have chosen as a test bed for our methodology is located on the campus of the University of Cape Town (UCT) and we make some remarks about this study context to assist later discussion.

Under apartheid, residential universities in South Africa were formally segregated by the Extension of Universities Act of 1959, and although some universities, including UCT, opposed and resisted this, they had very little success. By the mid-1960s South African universities were almost completely segregated along racial lines. The collapse of the apartheid government and the transition to democracy in 1994 reversed this, however, and by 2002, the demographic profile of UCT's student body was starkly changed. Of 18985 students, $52 \%$ were classified 'white' and $48 \%$ classified 'black' (a label that includes Africans, Indians and coloureds) (Ndebele, 2002). The university's enrolment statistics represent, on a macro level, a relatively high degree of racial integration. One might expect this to be reflected on a micro level within the spaces of social interaction around the university, for example, lecture theatres, sports centres, and student cafeterias. One area on the UCT campus which is particularly apt for study is a set of steps leading up to the Jameson Hall. This hall is in several ways the epicentre of the upper campus. Examinations are held inside it, as are university lectures of special significance, graduation ceremonies and student social events. It is almost exactly in the middle of the campus and large numbers of students gather in the summer months on the vast bank of steps that lead to its entrance. It is used on a daily basis in these months as a transitory leisure space, where one might eat lunch 
while enjoying the sunshine and view of the city, or perhaps meet and chat to friends or colleagues between lectures and tutorials. Its popularity in this regard theoretically attracts people from diverse racial groups, affording them the opportunity to interact and making it an interesting space for the study of natural contact.

We should note that our intention in this research was not merely to explore an observational method, but to explore the method in the context of a particular problem or phenomenon, namely intergroup contact and segregation in informal, dynamic spaces. We were specifically interested in whether its explicit preservation of spatiality and temporality would yield observational data that gave different or better insights into the nature of interaction and segregation in informal spaces.

\section{METHOD}

\section{Apparatus}

A Canon G2 3 megapixel digital camera was used to collect photographic images of the assemblage of students on Jameson steps. The camera was mounted on a tripod $22 \mathrm{~m}$ from the base of the steps. Measurements (height, width and breadth) were taken of steps, landings, relative position of the camera and other aspects of the physical space that were required for the construction of a three-dimensional model. Figure 1 depicts the observational situation.

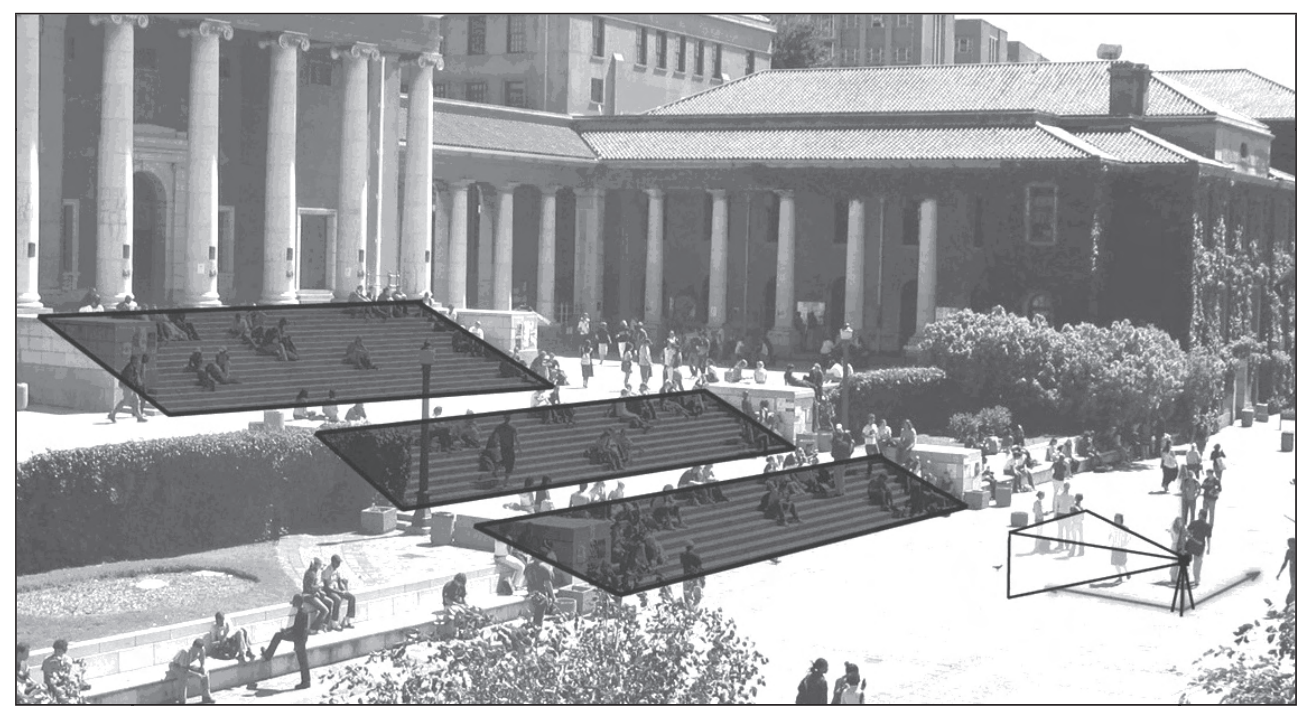

Figure 1. The observational situation, Jameson steps, University of Cape Town 


\section{Software}

In order to code information from digital photographs of the space more easily, we custom-designed a computer software program. This program allows a coder to work through a time sequence of photographs, marking the seating position of each person in the space, and recording his or her race and gender, as well as recording whether the individual arrived in a group or alone. A second software program transforms the two-dimensional observations to three dimensions, using measurement information about the physical nature of the space. Finally, a third program displays the observational data in three dimensions on a computer screen. This program allows the viewing perspective to be manipulated and shows the data in an animation, using a 1:30 time scale. Some examples of how the software works can be viewed at the Internet site http://www.uct.ac.za/depts/psychology/plato/contact.

\section{Participants/events}

Although we were interested in individual-level observations in this study, we collected data at an event level and, consequently, knew very little directly about participant attributes. We selected five different weekdays on which to collect observations (i.e., Monday to Friday), four of which were consecutive. The final observation day was delayed by seven days, due to rain. The period from 10:30 to 11:00 in the morning was chosen for each observation session. This choice of time period allowed us to observe fairly large numbers of people utilising the space, without it being so crowded that meaningful data could not be extracted. The full thirty-minute sessions were coded.

\section{Procedure}

The camera was set up on each of the observation days at a predetermined and constant location, in front of the Jameson steps. One high-resolution photograph $(2048 \mathrm{x}$ 1536 pixels) was taken every thirty seconds, for a total of half an hour on each occasion. This produced a total of 60 frames per session, and a total of 300 frames over the five days of observation. Exposure was increased marginally from the automatic reading given by the camera's built-in light meter, so as to achieve images in which it was easier to discern people and that were thus easier to code.

Images were downloaded in high-resolution .jpeg format, and were loaded onto a computer for coding. Three raters coded different subsets of the images, using bespoke software, which we described earlier. Raters were required to code the physical location of each person in the space, as well as each person's race, sex and 'unit identity'. This last-mentioned code represents whether the person entered the space alone, or as part of a group. An interrater reliability check was conducted on a subset of the images and the results of this check are discussed later.

Data were then transformed from the two dimensional points captured by coders to three-dimensional data, using additional bespoke software. This transformation depends on knowledge of the physical structure of the space, and is a fairly standard 
and well-known matrix operation (see Lang, 1987). We will not outline the details here, but will be glad to correspond with interested parties.

\section{RESULTS}

The central intention of this article is to outline an observational and analytic method for the study of contact and segregation in informal or leisure spaces, so that the spatial and temporal nature of the data is preserved. Accordingly, we are more interested in this section in ideas for analysing data that derive from the observational situation outlined in the earlier parts of this article. We do apply these ideas to data collected in the present study, though, and this produces some unanticipated results. The most surprising result is perhaps that the space is not populated in a fluid and shifting manner, as one might presuppose from the informal interactions enacted in it. Some of the ideas we suggest require relatively sophisticated techniques, but others depend on fairly ordinary and well-known descriptive and inferential statistical techniques.

\section{Descriptive statistics}

The total number of people entering the space in the time periods in question, and their breakdown into race and gender can be seen in Table 1. The descriptive data show that the majority of people, almost $73 \%$, who sit on Jameson steps are white. There is a noticeably low proportion of Asians, black and coloured people, but when their proportions are pooled into the other-than-white category, then this value rises to just over $27 \%$. Surprisingly, there was also quite a difference in the number of males on Jameson steps compared with females, roughly $63 \%$ and $37 \%$, respectively.

Table 1. Race and gender composition of people entering the study space

\begin{tabular}{|c|c|c|c|c|c|c|c|}
\hline \multicolumn{8}{|c|}{ Day } \\
\hline & 1 & 2 & 3 & 4 & 5 & Total & $\%$ of total \\
\hline Asian & 5 & 8 & 6 & 0 & 0 & 19 & 1.3 \\
\hline Black & 99 & 68 & 78 & 8 & 19 & 272 & 18.3 \\
\hline Coloured & 53 & 11 & 6 & 18 & 26 & 114 & 7.7 \\
\hline White & 314 & 298 & 236 & 135 & 97 & 1080 & 72.5 \\
\hline Male & 290 & 243 & 201 & 98 & 103 & 935 & 62.8 \\
\hline Female & 181 & 142 & 125 & 63 & 39 & 550 & 36.9 \\
\hline TOTAL & 471 & 385 & 326 & 166 & 142 & 1490 & \\
\hline
\end{tabular}

A chi-square test was applied to test how the sample's race and gender distribution compares with the UCT population as a whole. UCT census data from 2002 (see 
the Internet page at http://www.uct.ac.za/uct/docs/vc/vcreport2002.pdf) was used to determine the expected frequencies regarding race group.

The chi-square test for race yielded a significant result $\left(\chi^{2}=255, d f=1, p<\right.$ $0.001)$, indicating that the sample taken from Jameson steps is not representative of UCT as a whole but appears to over-represent white students and under-represent black, coloured, and Asian students. A similar test for gender was also significant $\left(\chi^{2}=84.8, d f=1, p<0.001\right)$, implying that males are over-represented and females under-represented.

\section{Intercoder reliability}

As stated previously, custom software was designed to enable precision in locating individuals in the space in question and, in fact, the resolution at which this is possible with the software is far higher than required. We therefore did not deem it necessary to test the interrater reliability of the location coding. However, the coding software required the manual assignment of race and gender, and we decided to test this assignment for coding errors. A small sample, $5 \%$ of the data, was coded for race and gender by two independent raters. The raters agreed on $93.3 \%$ of race assignments, and on $100 \%$ of gender assignments. ${ }^{2}$

\section{Cross-tabulations for examining racial homogeneity and heterogeneity}

People entering or sitting in the space were coded for whether they belonged to a social group or not. (Some people entered the space in a group, whereas others entered as individuals, but joined a group, or were joined by a group, and still others entered and remained as individuals.) We coded groups for whether they were racially heterogeneous or homogeneous, and Table 2 shows the proportions for each of these per day and over the total period. We again show a similar coding for gender, in order to give

Table 2. Racial and gender homogeneity and heterogeneity in groups occupying the study space

\begin{tabular}{|c|c|c|c|c|c|c|c|}
\hline & Mon & Tues & Wed & Thur & Fri & Total & $\%$ \\
\hline Total & 109 & 100 & 83 & 48 & 53 & 393 & \\
\hline \multicolumn{8}{|l|}{ Race } \\
\hline Homogeneous & 79 & 84 & 62 & 46 & 47 & 318 & 80.9 \\
\hline Heterogeneous & 30 & 16 & 21 & 2 & 6 & 75 & 19.1 \\
\hline \multicolumn{8}{|l|}{ Gender } \\
\hline Homogeneous & 52 & 61 & 49 & 26 & 34 & 222 & 56.5 \\
\hline Heterogeneous & 57 & 39 & 34 & 22 & 19 & 171 & 43.5 \\
\hline
\end{tabular}


a point of comparison. In our analysis, only those groups that consisted of more than one person were considered, since a group consisting of one member is by definition homogeneous and so its inclusion would bias the results towards seeing the space as more segregated than it really is.

The data indicate that racially mixed groups are relatively scarce in the space, namely $19.1 \%$, whereas groups composed across gender lines are quite common, namely $44 \%$. Chi-square tests employed to test for consistency of this pattern over the five days were significant for both race and gender $\left(\chi^{2}=16.71, d f=4, p<0.002\right.$; and $\chi^{2}=161.16, d f=4, p<0.002$ ), showing that the distribution pattern of heterogeneous and homogeneous groups varied over the five days. It is clear from the data, though, that it is always relatively rare to encounter heterogeneous race groups on the Jameson steps.

\section{Preserving temporality: analyses of segregation and integration}

Analyses that immediately suggest themselves in respect of assessing segregation and integration in the space in question are those commonly used in urban geography and sociology to chart residential segregation. Massey and Denton (1988) provide a detailed discussion of these, and of the corresponding measures.

The dissimilarity index (D) is widely used to reflect the evenness in distribution of two race groups over a set of spaces (usually suburbs). The index yields scores that range from a minimum of 0 (complete integration) to a maximum of 1 (complete segregation). This measure destroys the spatiality of segregation/integration, though - we need to artificially segment our space into sub-spaces in order to use it at all - and is therefore only partly useful for our purposes. Also, there is no known sampling distribution for $\mathrm{D}$ and tests of statistical significance have to be conducted through Monte Carlo simulations.

The interaction index (xPy) is a measure of potential exposure (Massey \& Denton, 1988); in other words, it measures the probability of a member from one social group interacting with a member from a different social group to his or her own. It also yields a score between 0 and 1, with higher values signalling a greater probability that inter-group contact will take place (Massey \& Denton, 1988).

Since the last-mentioned two indices were designed to measure residential segregation across aerial units, their use in the present study required the artificial division of Jameson steps into regions. We identified six zones on the basis of three landings and a line of vertical symmetry. The indices were calculated according to the two groups, 'white' and 'other-than-white', measuring evenness and exposure (two different measures of segregation) across the six afore-mentioned zones at various time intervals. Figure 2 shows the layout required to allow the calculation of D and xPy for our data.

Partitioning Jameson steps into six regions allowed us to employ standard segregation indices, but it also permitted the use of a chi-square test for equality of racial 


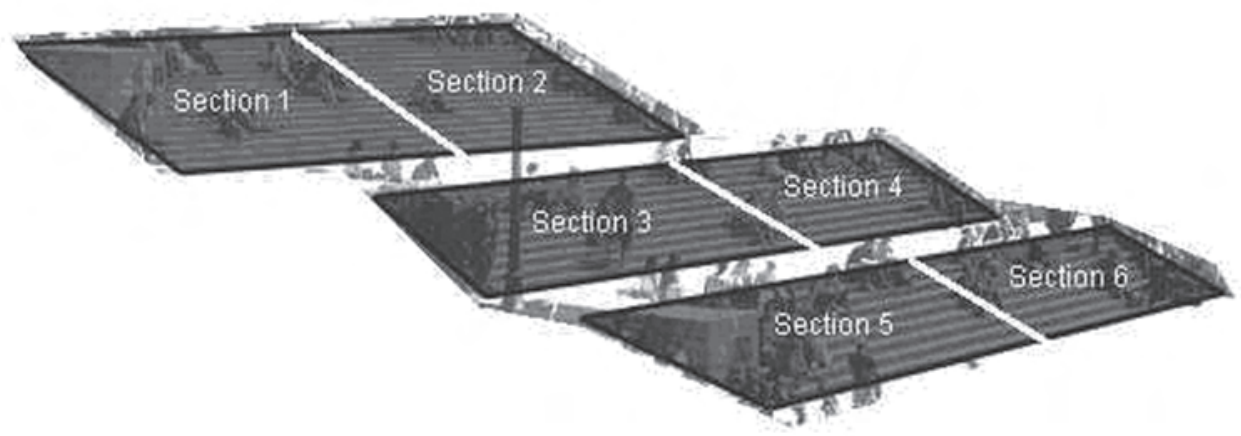

Figure 2. Partitioning of the step space to allow calculation of the segregation indices, D and $\mathrm{xPy}$

distribution across sessions. By combining the frequencies of each session we produced a contingency table describing racial distribution over five days, allowing a test of whether the space is populated in a similar fashion over all the days. If race groups do not prefer particular regions in the space consistently over time, then the act of summating the frequencies should equalise the distribution of races across regions. A significant chi-square test for equality of distribution, on the other hand, suggests racialised seating patterns that are not manifested on a given day but apply more broadly, implying a predetermined informal structure to the space. Table 3 shows the cross-tabulation in question, with the omission of 'Asians' due to their extremely low proportion (0.005) in the space. A chi-square test for equality of distribution was significant $\left(\chi^{2}=50.73, d f=10, p<0.001\right)$, indicating that there does appear to be a consistent racialised distribution in the study space. Inspection of the contingency table suggests that black students tend to predominate in Regions 1 and 2, whereas white students tend to avoid sitting in Region 1, and coloured students avoid Regions 5 and 6.

Table 3. Summated racial distribution across regions in the space for two days

\begin{tabular}{|lrrrrrll|}
\hline \multicolumn{7}{c|}{ Region } \\
\hline White & 1 & 2 & 3 & 4 & 5 & 6 & TOTAL \\
Coloured & 17 & 49 & 47 & 56 & 64 & 43 & 276 \\
Black & 6 & 8 & 7 & 11 & 1 & 2 & 35 \\
TOTAL & 18 & 21 & 7 & 7 & 4 & 10 & 67 \\
& 41 & 78 & 61 & 74 & 69 & 55 & 378 \\
\hline
\end{tabular}

Our primary theoretical concern in this article was to find observational and analytic methods that preserve the temporality and spatiality of patterns of contact between race groups. Partitioning the space into six regions corrupts spatiality to some 
extent, but it is possible to preserve the temporality of our data, and examine patterns of integration and segregation in our data. We mentioned earlier that the measures of Dissimilarity (D) and Exposure (xPy) are two standards in urban georgaphy for assessing levels and segregation, and we calculated these for the six-level partition we have created, for each time unit and for two of the five days in question. (We do not display the data for the other days, as our intention here is primarily to consider analytic possibilities, not to provide an exhaustive analysis.)

The time scale considered in our analysis here is the most detailed to us, namely thirty seconds, which is a rather short time interval for the study of patterns of segregation, but may be appropriate for the high rate of flux in a dynamic social space. At the thirty-second time scale there is a high degree of fluctuation in segregation as measured by $\mathrm{D}$, although most of the data points lie in the range of 0.3 to 0.7 , with an average of 0.47 , and a standard deviation of 0.14 . Figure 3 displays the time sequences, and shows a trend towards decreasing segregation over time (which is apparent on each of the two days under consideration, suggesting that it is not a chance pattern but a consistent property of the space at that time of day). The data complexity has been reduced by averaging every third point (smoothing by moving average), making it easier to see the overall trend.

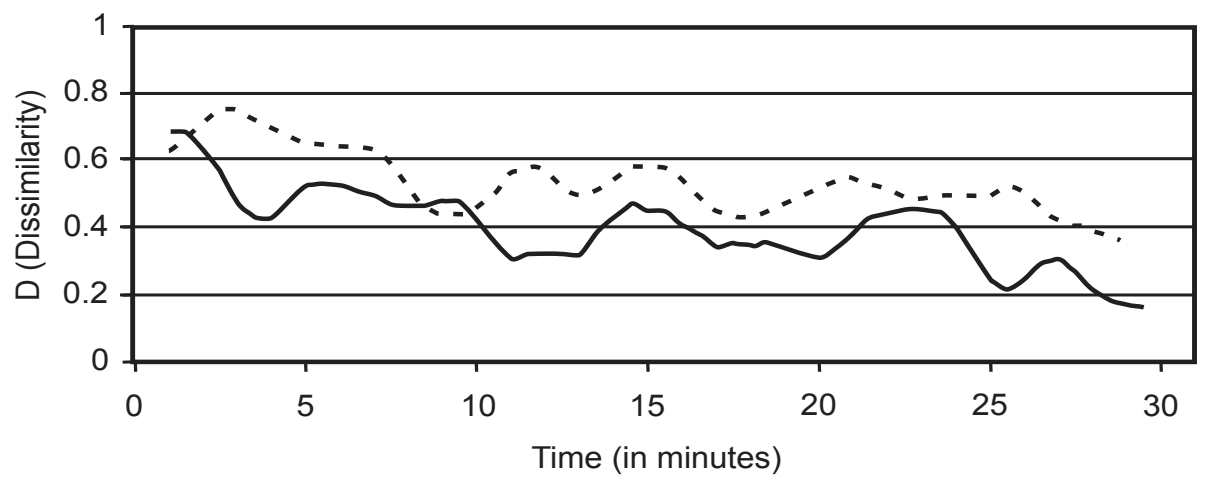

Note: The lines represent data for two different days and have been smoothed using a three-point moving average technique.

Figure 3. Variations in the dissimilarity index over 30 minutes for two days

Segregation as measured by the interaction index at a 30 -second interval shows a similar pattern to that for $\mathrm{D}$, with integration increasing over time for both sessions of observation. Figure 4 is a graphic depiction of the data.

Since time cannot itself be the mechanism underlying segregation, we wished to explore whether the study space was filling up over the time period in question, and whether this was associated with the decreasing segregation. We thus computed the 
correlation between each of the indices and the number of observations in each frame, for each day. The correlations between $\mathrm{D}$ and the (population) density of the space were -0.73 and -0.84 for each of the two days, respectively. Both these values are statistically significant $(p<0.001, d f=58)$. The correlations between the exposure index and the density of the space were 0.93 and 0.58 , respectively, and these are also statistically significant $(p<0.001, d f=58)$.

This analysis of dissimilarity and exposure reveals three important things. In the first place, the space we have chosen to study exhibits a clearly racialised seating pattern. Black, white and coloured students prefer to sit in different parts of the space, and they exercise this preference on each of the days we examined. More significantly, this preference is replicated over different days and by different students. Even though the space is informal and easily rearrangeable, students of a particular race group prefer to sit in the same area across different days. Finally, our conjecture about the temporality of inter-group contact is upheld by this analysis - Figures 3 and 4 clearly show how a 'snapshot' of the pattern of segregation based on the final photographic frame would miss the way in which this pattern evolved.

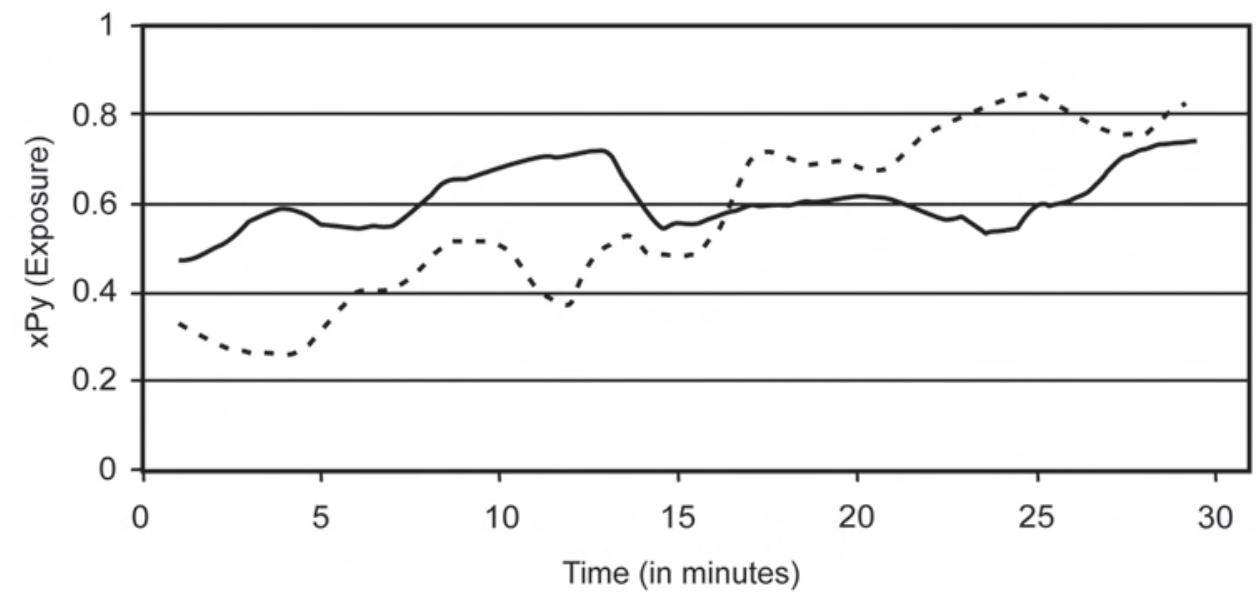

Note: The lines represent data for two different days, and have been smoothed using a three-point moving average technique.

Figure 4. Variations in the exposure index over 30 minutes for two days

\section{Preserving spatiality: Analyses of distance between race groups in the space}

Thus far, we have provided ideas and examples for analysis of naturalistic observations of intergroup contact. Some of these analyses preserve the temporality of the data, but all discussed thus far corrupt the spatiality of the original interactions. The method 
we have developed and wish to promote, on the other hand, attempts to preserve both the temporality and spatiality of observational data. Specifically, the data recovered from the coding and transformation of the photographic images take the form of a 7-tuple, where the first four components record the individuality, race, sex and group identity of each person in the space, and the final three components record the location of each person in three-dimensional physical space (relative to the 'window' on Jameson steps created by the camera's point of view).

As far as analysis of these data is concerned, we think that the most fruitful quantitative methods are not inferential but descriptive. To this end, we have customised two separate sets of software that allow for the animation of the three-dimensional data. In the first of these, the original three-dimensional data are preserved at the individual level, and the animation shows individuals leaving and entering the space. Data points can be colour-coded to show race or gender characteristics. In the second, points are treated as inherently race or gender characteristic, and the resolution is set around each individual point so as to move from the individual to the group level. Both pieces of animation software can be controlled at the level of single frames. These animations are not analytic 'end-points', but data simplification tools that may help in the generation of conjectures about the processes operating in the space.

Ultimately, the best way for a reader to evaluate the utility of the animation tools is to view them in action. Animation movies showing examples of analyses created by these programmes are available for viewing at the following Internet site: http://www. uct.ac.za/depts/psychology/plato/contact.html. They are .gif files, so most Internet browsers (e.g., Internet Explorer, Mozilla Firefox and Apple Safari) will load them seamlessly.

Figure 5 shows two frames from one of our animations. In the figure, black, white and coloured people are shown by differently shaded circles. The steps are shown as a two dimensional projection, the vertical axis of the figure capturing the height dimension of the physical layout. Black people are seen to congregate towards the top of the steps, and white people in the middle and bottom. This spatial pattern is evident in both frames, as it indeed is over different days.

It is also possible to inferentially test specific conjectures about processes of segregation and integration in the space represented by the three-dimensional data. One hypothesis we entertained, for instance, was that initial states of segregation within the space would determine later states. Thus, a person entering the space at time ${ }^{\mathrm{k}+1}$ is entering a space that has already been partitioned by prior occupants, most immediately at time ${ }^{k}$. More specifically, if collectives of people tend to maintain or increase levels of intergroup segregation in such a space, then each new person (or group of persons) entering the space will prefer to sit closest to a member of their own group. The null hypothesis that must be refuted in order to test this conjecture is that seating choices will be random with respect to the race of the nearest neighbour in the space. The number of white students who select nearest neighbours who are also white can 
be collected, and a binomial distribution can be used to estimate the probability that this happened, given an assumption of randomness. When this method is applied to the data we collected over five days, we find that white students show a strong tendency to join areas that are populated by other white students (407 of 740), but this pattern

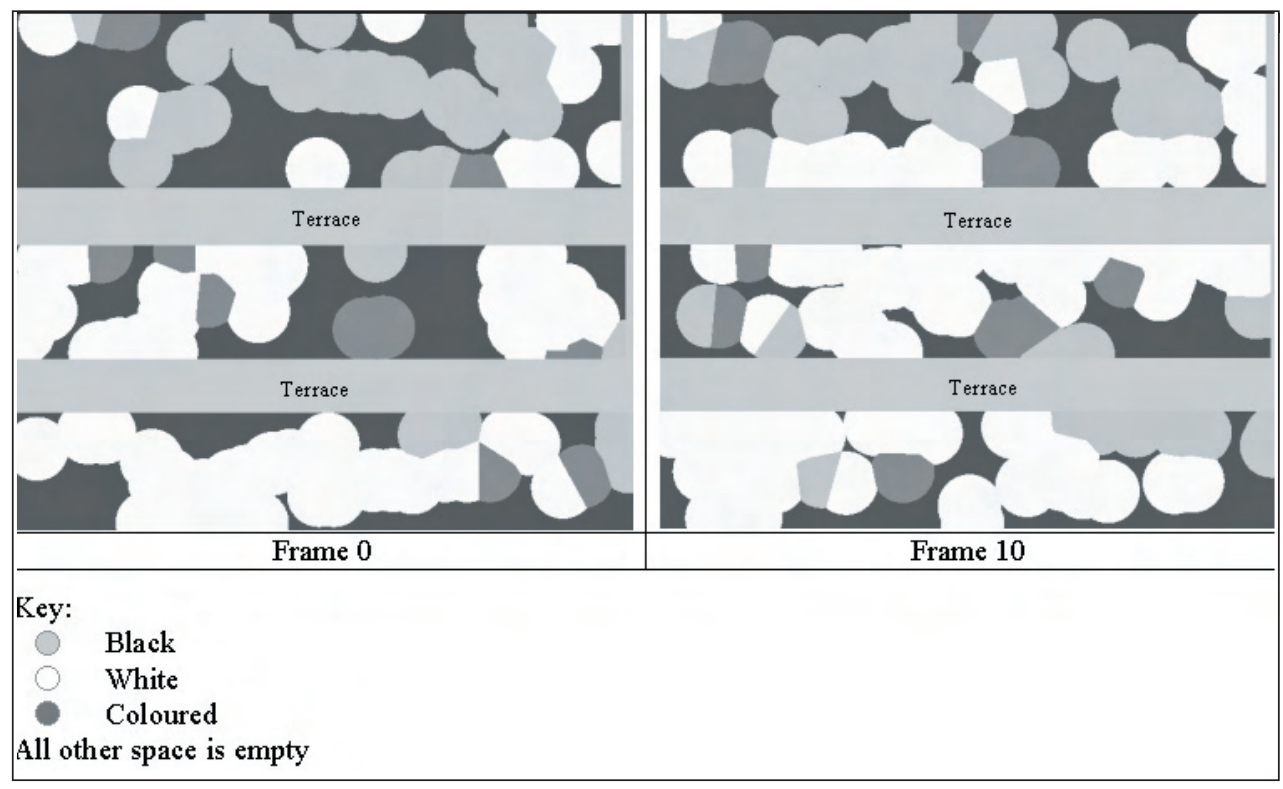

Figure 5. Examples of animation frames, showing spatial pattern of segregation on steps

is not shown by black students (47 of 177). Binomial probabilities calculated for these frequencies, assuming three possible seating choices ('own race' space, 'other race' space and neutral), indicated that the pattern for white students was unlikely to be due to random seating choices $(\mathrm{p}<0.001)$, but for black students, the pattern was compatible with a random seating choice $(\mathrm{p}>0.6)$.

\section{DISCUSSION}

In this article, we have proposed a method for enhancing the naturalistic study of contact between race groups. We support the claim that it is important to understand and theorise the spatiality of intergroup contact (e.g. Dixon, 2001), but we propose that it is also important to study the temporality of intergroup contact. The observational and analytic methods we set out in this article thus aim to preserve both the spatiality and temporality of contact data.

The preservation of temporality is not a mimetic strategy in our scheme, but a route to a better understanding of the ways in which groups interact. Contact unfolds 
as a process, and static views of intergroup behaviour may misrepresent the nature of interactions. We argued earlier, following Schelling (1978), that what appears to be an integrated seating pattern, that is, one in which participants do not appear to show a preference for sitting near to members of their own group, may be the outcome of a segregative process. Schelling used mathematical simulations (and a dose of common sense) to make this point, but provided no data to show that the phenomenon occurs in naturalistic settings. We have provided data in this article of precisely such a phenomenon. Students occupy a public space on their campus, and when the space is relatively empty they self-segregate. However, when the space fills up, and there is competition for seats (or less choice) the seating pattern becomes less segregated. This finding is not consistent with some previous literature. For example, Davis, Seibert and Breed (1966) documented the diametrically opposite pattern, in which segregation on public transit increased as the bus filled up. However, that study was conducted at a time in New Orleans just after the desegregation of buses and there are some very specific aspects of the way spatiality was controlled in the buses in question that make comparison difficult.

This is one demonstration of the importance of studying inter-group contact in terms of a time-contiguous sequence of events, but we believe that we have unearthed several others. One important phenomenon may be the dynamic nature of public spaces: the state the space starts in, for instance, may determine or influence later states. Thus, existing states of segregation or integration in a public space may be expected to influence the seating choice new arrivals make. On entering the space, a seating pattern is observed, and this provides the observer with an obvious and easily assimilated choice. This pattern may reflect wider, macro-social patterns, but it need not. The point is that prior states of a space can be expected - to some extent - to have independent effects on later states, and this may be partly how patterns of segregation are replicated in social spaces. The interesting thing to note is that attitudinal elements need not be present at all in the unfolding of a spatial and temporal pattern. We suggest in our analysis of the data that we do indeed see a self-replicating pattern in the campus space we observed. White students at time $e_{k}$ made seating choices that mapped onto the racialised seating pattern at time $\mathrm{k}_{\mathrm{k}-1}$ at a level well above what one would expect by chance variation alone. The same was not true of black students, though this might be due to the fact that this group was less densely represented (by a factor of three) in the space.

The observational method we propose and explore in this article may appear to be relatively complicated from a technological point of view, and one may wonder whether the advantages it confers justify the effort required in setting up the camera equipment and software. In fact, we believe that this is relatively straightforward, and that the software tools we have devised simplify the technology sufficiently to allow its use by any social psychological researcher. Although we used a 3.3 megapixel 
camera, 6 and 7 megapixel cameras are now quite affordable and better results can be expected from these higher-resolution models. The bottleneck is likely to come when coding the two-dimensional data, but any modern desktop computer equipped with sufficient random access memory (RAM) and a reasonably powerful processor (e.g. Pentium 4, Apple G5) should suffice.

The data captured using our observational method are individual-level data but it should be said that very little information is returned from our coding regarding each individual. We can locate and track each person's position in the space, over time, and we can make reasonably accurate estimations of race and sex, and to which group in the space they belong. However, we cannot say anything further about the individual (e.g., from a psychological, attitudinal or conversational point of view), and we suspect that this limitation is inherent to our method. It is of course possible to investigate the role of say, social representations, in the seating decisions students make in the space in question, but we do not see an easy way of tying this information to the observational data.

There are, of course, many different levels on which one can analyse the phenomenon of racial segregation. Results from the present study suggest that, in the context of one particular university (with a liberal reputation, and an integrated student populace, at least from demographic statistics), there is segregation in respect of seating choice, with different race groups preferring to occupy different areas within an accessible public space. The space in question (Jameson steps, at UCT) tends to be used more by white students and less by students from other groups, relative to their respective proportions at the university as a whole. This finding is consistent with previous research conducted in an educational setting (Sagar, Schofield \& Snyder, 1983; Schofield \& Francis, 1982; Schofield \& Sagar, 1977).

The finding that the space under study shows an uneven distribution along racial lines across five days of observation suggests that the space is not as 'fluid' as one might assume at first glance. It also reinforces, once again, the importance of taking temporality into account when studying patterns of contact. In this case, the time window is 24 hours, rather than the 30 seconds we allowed between photographic frames. A static snapshot taken at one point in time would have missed the consistency of segregation across days, just as it would have missed the variation in segregation within a half-hour period. We found that certain regions in the space are consistently preferred by race groups, which seems to imply a process of spatial organisation at an inter-group level. One could argue that this merely reflects habitual seating tendencies of people (in the same way that university staff might consistently choose the same seat in the staff room) but the counter-argument is then to ask why it occurs across racial lines every time? One explanation might be that there is a shared understanding amongst members of particular racial groups regarding 'their' place on Jameson steps, but we do not have any evidence beyond our observational data that would support this claim. 
The particular space we chose to study of necessity has imposed many limitations on the kinds of conclusions we are able to draw. It is only one informal space that students use on campus, and a study of the entire set of informal spaces would undoubtedly give us a more representative view of contact and segregation on campus. However, this could not have been achieved without changing the overall methodology of the study, and this would have imposed other limitations that the present method was designed to overcome. The methodology we chose to use is precisely the point of the present article.

Another limitation of the study may also be an over-reliance upon quantitative data, and it is clear that interview data may have served to enrich our understanding of contact and segregation processes on campus, and in that particular space, but it would have taken the present piece way beyond its intended focus. There is certainly scope (and need) for such a study, perhaps of an ethnographic nature, and this might shed light on the lived nature of the space. A reviewer of this article has speculated that there may be a shared social representation of the space on the UCT campus as 'owned' by white males, and other students may have self-segregated in spaces they have claimed as their 'own'. These are precisely the possibilities that an ethnographic analysis may be able to uncover and that are beyond the reach of observational methodologies.

In summary, the present study was intended as a demonstration of a starting point for a way of studying informal segregation that preserves both space and time processes, taking advantage of recent advances in photography and computer technology. We believe that this approach has great merit, and that our experimentation with the approach in a particular space on a university campus has shown this to be the case.

\section{ACKNOWLEDGEMENTS}

The National Research Foundation (NRF) of South Africa, ESRC of England, and the South Africa - Netherlands Research Programmeme on Alternatives in Development (SANPAD) of the Netherlands (Grant 02/21), provided support to the first, second and fifth authors for the research reported in this article.

\section{NOTE}

1 Aspects of the present research derive from an Honours research project conducted by Stephen Underwood at the University of Cape Town in 2002, under supervision of the first and fifth authors.

2 A reviewer has raised some concerns about how race was coded in this study. Specifically, we have no way of knowing whether the coding made by raters (although high in interrater agreement) corresponded with the categories that the people in the space would have assigned themselves. We concede this point. 


\section{REFERENCES}

Campbell, D. T., Kruskal, W. H. and Wallace, W. P. (1966). Seating aggregation as an index of attitude. Sociometry, 29, 1-15.

Christopher, A. J. (1991). The atlas of apartheid. London: Routledge.

Davis, M., Seibert, R. and Breed, W. (1966). Interracial seating patterns on New Orleans public transit. Social Problems, 13, 298-306.

Dixon, J. A. (2001). Contact and boundaries 'locating' the social psychology of inter-group relations. Theory and Psychology, 11, 587-608.

Dixon, J. A. and Durrheim, K. (2003). Contact and the ecology of racial division: Some varieties of informal segregation. British Journal of Social Psychology, 42, 1-23.

Dixon, J. A. and Reicher, S. (1997). Inter-group contact and desegregation in the new South Africa. British Journal of Social Psychology, 36, 361-381.

Durrheim, K., and Dixon, J. A. (2001). The role of place and metaphor in racial exclusion: South Africa's beaches as sites of shifting racialization. Ethnic and Racial Studies, 24, 433-50.

Goldberg, D. T. (1993). Racist culture: Philosophy and the politics of meaning. Oxford: Blackwell.

-. (1998). The new segregation. Race and Society, 1, 15-32.

Henderson-King, E. (1994). Minimizing inter-group contact: An urban field study. Journal of Applied Social Psychology, 24, 1428-1432.

Jahoda, M. and West, P. S. (1951). Race relations in public housing. Journal of Social Issues, 7, 132-139.

Lang, S. (1987). Linear algebra. New York: Springer Verlag

Massey, D. and Denton, N. A. (1988). The dimensions of residential segregation. Social Forces, 67, 281-315.

McCauley, C., Plummer, M., Moskalenko, S., and Mordkoff, J. B. (2001). The exposure index: A measure of intergroup contact. Peace and Conflict: Journal of Peace Psychology, 7, 321-336.

Ndebele, N. (2002). Vice-Chancellor's report, 2002. http://www.uct.ac.za/uct/docs/vc/ vcreport2002.pdf. (accessed on 24 January 2005).

Pettigrew, T. F. (1998). Inter-group contact theory. Annual Review of Psychology, 49, 65-85.

Pettigrew, T. F. and Tropp, L. R. (2000). Does intergroup contact reduce prejudice? Recent meta-analytic findings. In S. Oskamp (Ed.), Reducing prejudice and discrimination: The Claremont Symposium on applied social psychology (pp. 93-114). Mahwah, NJ: Erlbaum.

Philipp, S. (2000). Race and the pursuit of happiness. Journal of Leisure Research, 32, 121-124.

Robinson, J. L. Jr. (1980). Physical distance and racial attitudes: A further examination of the contact hypothesis. Phylon, 41, 325-332.

Rogers, M., Hennigan, K., Bowman, C. and Miller, N. (1984). Inter-group acceptance in classrooms and playground settings. In N. Miller and M. Brewer (Eds), Groups in contact: The psychology of desegregation (pp. 213-227). New York: Academic Press.

Sagar, H. A., Schofield, J. W. and Snyder, H. N. (1983). Race and gender barriers: Preadolescent peer behaviour in academic classrooms. Child Development, 54, 1032-1040. 
Schelling, T. C. (1978). Micromotives and macrobehaviour. New York, London : W.W. Norton and Company.

Schofield, J. W. and Francis, W. D. (1982). An observational study of peer interaction in racially mixed 'accelerated' classrooms. Journal of Educational Psychology, 74, 722-732.

Schofield, J. W. and Sagar, H. A. (1977). Peer interaction patterns in an integrated middle school. Sociometry, 40, 130-138.

Stephan, W. G. (1987). The contact hypothesis in inter-group relations. Review of Personality and Social Psychology, 9, 13-40.

Taylor, D. M. and Mogghadam, F. M. (1996). Theories of intergroup relations (2nd Ed.). Westport: Praeger 